DERECHO COMPARADO EN INVESTIGACIÓN Y ENSEÑANZA DE DERECHO EN CARRERAS ECONÓMICO ADMINISTRATIVAS.

\title{
DERECHO COMPARADO EN INVESTIGACIÓN Y ENSEÑANZA DE DERECHO EN CARRERAS ECONÓMICO ADMINISTRATIVAS
}

\section{COMPARATIVE LAW IN RESEARCH AND TEACHING IN ECONOMIC-ADMINISTRATIVES PREGRADE PROGRAMS}

José Armando Flores Sánchez*, Ricardo Maglioni Montalvo**

\begin{abstract}
* Doctor en Estudios Jurídicos. Profesor Investigador de la Universidad Juárez Autónoma de Tabasco de la División Académica de Ciencias Económico Administrativas. ORCID: http://orcid.org/0000-0003-3015$787 \mathrm{X}$.

** Doctor en Derecho. Profesor Investigador de la Universidad Juárez Autónoma de Tabasco de la División Académica de Ciencias Económico Administrativas. Email: rmaglioni23@hotmail.com. ORCID: http://orcid.org/0000-0001-5733-3453.
\end{abstract}

Dirección para recibir correspondencia: doctorpnpc@gmail.com

Fecha de recibido: 15 de enero de 2019 
DERECHO COMPARADO EN INVESTIGACIÓN Y ENSEÑANZA DE DERECHO EN CARRERAS ECONÓMICO ADMINISTRATIVAS.

\section{RESUMEN}

Este trabajo, describe el Derecho Comparado como una de las metodologías contemporáneas que se emplean al llevar a cabo investigaciones vinculadas al derecho, en las carreras de Contabilidad, Mercadotecnia, Economía, Administración, entre otras.

OBJETIVO: Describir el método de investigación de Derecho Comparado para que estudiantes o profesores universitarios realicen investigaciones en Derecho, considerando este método.

MATERIAL Y MÉTODO: Utilizando como instrumento de investigación el cuestionario con preguntas abiertas, por medio de la doctrina analítica se describen el método de estudio del Derecho Comparado.

RESULTADOS: Al entrevistar a profesores que imparten materias del área de Derecho en Licenciaturas de Contaduría Pública, Administración, Economía o Mercadotecnia, los métodos que más conocen o utilizan son: Derecho Comparado, Estudio de Caso, Análisis Económico del Derecho, Historia Crítica y Doctrina Analítica. Encontrando también que son pocos los estudiantes que deciden titularse por medio de investigaciones que concluya en publicación de artículos o tesis.

CONCLUSIONES: Son pocos los estudiantes de nivel licenciatura que se interesan en realizar investigaciones de su área, de 300 estudiantes, solo seis se titulan por artículos científicos, 17 por tesis, y solo uno por resolución de problemas o casos prácticos. La gran mayoría no realiza investigaciones por no saber efectuarlas.

PALABRAS CLAVE: Método contemporáneo. Estudio del Derecho. Estudio de caso. Derecho Comparado. Enseñanza-aprendizaje. 
DERECHO COMPARADO EN INVESTIGACIÓN Y ENSEÑANZA DE DERECHO EN CARRERAS ECONÓMICO ADMINISTRATIVAS.

\section{ABSTRACT}

This article describes Comparative Law as one of the contemporary methodologies used in law related research in accounting, marketing, economics, administration, and among other knowledge areas.

OBJECTIVE: To describe the research method in comparative law so college students and faculty do research, using this method.

MATERIAL AND METHOD: An open-question instrument was used in this research, and the study method of comparative law is described through analytical doctrine.

RESULTS: Law faculty currently teaching law, in accounting, administration, economics and marketing pregrade programs, was interviewed. The methods used by the interviewed faculty are: comparative law, study case, economic analysis of law, critical history and analytical doctrine. It was also found that students prefer to gradute under the thesis as well as the scientific article procedures.

CONCLUSIONS: Few students are interested in doing research, out of 300 students, six of them choose to graduate by writing a scientific article, 17 prefer to do a thesis and only one by practical cases or problem resolutions. While the others do not do research due to the fact that they do not know how to.

KEY WORDS: Contemporary method. Law study. Study case. Comparative law. TeachingLearning.

\section{INTRODUCCIÓN}

El estudio de leyes es de importancia en nuestra sociedad desde cualquier punto de vista; por ello, el presente trabajo tiene como objetivo hacer un análisis de los métodos contemporáneos de estudio y enseñanza del Derecho, que bien pueden ser empleados tanto en la instrucción de los estudiantes que cursan la carrera de leyes, o los que estudian materias de Derecho en Licenciaturas de Contaduría Pública, Administración y Economía. 
DERECHO COMPARADO EN INVESTIGACIÓN Y ENSEÑANZA DE DERECHO EN CARRERAS ECONÓMICO ADMINISTRATIVAS.

Los estudiantes de licenciaturas, no cursando la carrera de Derecho, dentro de su programa de estudio, tienen que aprobar materias como Derecho Laboral, Civil, Administrativo, entre otras y es importante desarrollar el proceso enseñanza-aprendizaje con métodos actuales que permitan formar profesionales, conocedores de la teoría y práctica de las asignaturas en cuestión.

Es importante notar, lo que hoy es verdad, no lo fue muchos años atrás, como la veracidad que estaba en contra de Galileo en el siglo XV, quien afirmaba que la tierra se movía, o como menciona Karl Popper, refiriéndose a Ptolomeo, quien en su tiempo creía que la tierra era el centro del universo, o el mismo Newton que se equivocó al decir que el sol era el centro del universo y hoy se cree que el universo carece de centro (Popper, 2010, pp. 327-328); por lo tanto, la verdad que analizamos hoy, no será la misma en los años venideros.

En México, la enseñanza tiene libertad de cátedra, de acuerdo a lo que establece la fracción VII del artículo tercero de la Constitución Política de los Estados Unidos Mexicanos (CPEUM) que a la letra establece:

Las universidades y las demás Instituciones de Educación Superior a las que la ley otorgue autonomía, tendrán la facultad y la responsabilidad de gobernarse a sí mismas; realizarán sus fines de educar, investigar y difundir la cultura de acuerdo con los principios de este artículo, respetando la libertad de cátedra e investigación y de libre examen y discusión de las ideas; determinarán sus planes y programas; fijarán los términos de ingreso, promoción y permanencia de su personal académico; y administrarán su patrimonio.

En esa libertad, se debe buscar la mejor manera de enseñar y educar, una de esas maneras es la investigación pertinente, procurando que esa investigación se difunda y aporte a las generaciones presentes y futuras de nuestro país, en virtud de que el mundo globalizado y la competencia requiere cada día profesionistas más preparados y técnicos.

El método utilizado en este artículo es el estudio jurídico de doctrina analítica; para tal efecto, se realiza una investigación teórica y empírica, empleando como instrumento de indagación el cuestionario con preguntas abiertas. 
DERECHO COMPARADO EN INVESTIGACIÓN Y ENSEÑANZA DE DERECHO EN CARRERAS ECONÓMICO ADMINISTRATIVAS.

Este fue aplicado de forma aleatoria a los profesores que imparten materias en las Licenciaturas de Contaduría Pública, Administración, Economía y Mercadotecnia en la Universidad Juárez Autónoma de Tabasco (Población analizada).

Cabe mencionar que, las investigaciones actuales de cualquier tipo y en diferentes campos, demanda investigaciones inter y multidisciplinarias preferentemente mixtas.

La enseñanza del Derecho, como cualquier asignatura, requiere de académicos preparados y comprometidos, que se ajusten con las exigencias programáticas de la modernidad líquida de la que habla Baumman (2015), "siendo el derecho una inusual combinación de lo académico y lo profesional" (Owen, 1999, p. 25).

Por ello, es importante señalar que en la actualidad, muchos profesores de las materias de Derecho combinan esa práctica con el ejercicio profesional; es decir, tienen sus despachos particulares donde son abogados postulantes o notarios públicos.

Se realizó una encuesta a profesores, quienes coincidieron que pocos son los estudiantes de licenciaturas que realizan investigaciones; dado que, según los docentes, la gran mayoría desconoce la forma correcta de citar la aportación de los autores o no está familiarizado con los diferentes estilos de citas bibliográficas.

Como profesor investigador, se ha encontrado que los estilos de cita más utilizados son:

- APA American Psychological Association: utilizado mayormente en Psicología;

- Manual de Estilo Chicago;

- Uniform (antiguo Vancouver) utilizado en el ámbito de la salud;

- Harvard (autor y fecha: utilizado en Física, Ciencias naturales y Ciencias sociales);

- Existen otros tipos de citaciones establecidas, por ejemplo, el Instituto de Investigaciones Jurídicas de la UNAM, y diferentes revistas que plantean su estilo en cita.

En este sentido, los catedráticos utilizan diferentes métodos de investigación en el área de Derecho, entre los que destacan: Derecho Comparado, Estudio de Caso, Análisis Económico del Derecho, Historia Crítica, Doctrina Analítica, Sociología Jurídica, Política Jurídica, y la forma y metodología de enseñanza se van perfeccionando en las universidades públicas y privadas; razón por la que en este artículo se resalta el método de estudio de Derecho Comparado, pues 
DERECHO COMPARADO EN INVESTIGACIÓN Y ENSEÑANZA DE DERECHO EN CARRERAS ECONÓMICO ADMINISTRATIVAS.

se considera que los estudios en otras latitudes pueden aportar mucho en las investigaciones en el área de derecho.

\section{MÉTODO DE ESTUDIO DE DERECHO COMPARADO}

Las exigencias de la modernidad, dado que vivimos en un mundo globalizado (Baumman, 2017) nos impulsan a prepararse mejor cada día. "Las maneras de ver hoy el derecho demandan una visión integral del fenómeno jurídico" (González, 1998, p.15). Por tal motivo, el "profesor universitario, o bien el investigador, como todo ser humano, debe utilizar los elementos a su disposición para alcanzar sus objetivos" (González, 1998, p. 14).

Bömher (1999) realiza un interesante comentario en relación a la enseñanza del Derecho, respecto de que "si el profesor se dedica sólo a la enseñanza, o si la realiza como tarea subordinada de la principal" (p. 16). Lo que no se puede negar es, que el profesional que enseña Derecho o cualquier otra asignatura, además del conocimiento, es prudente que conozca de la práctica y aplique esos conocimientos a resolver problemas reales, y debe hacerlo por medios de métodos, objetivo principal de este trabajo.

Agrega también el mismo autor que "La enseñanza del derecho se convierte, entonces, en una forma bien directa de incidir en las políticas públicas de nuestras naciones y, por lo tanto, debería formar parte de cualquier agenda seria de reformas institucionales" (Böhmer, 1999, p. 16).

El maestro Jorge Witker (2013) inicia su estudio sobre la enseñanza del Derecho aseverando que "este escenario del fin de siglo ha sido impactado de nuevos paradigmas e instituciones, $y$ que debemos recrear nuestra cultura y abrir la mente a espacios zonales y globalizados que nos exigen esfuerzos de estudio e imaginación" (pp. 11-12).

Por otro lado, Carlos Eisenmann (2013) afirma que:

En la organización de la enseñanza del Derecho, como en cualquier otra instrucción, el primer problema que se presenta es el determinar sus fines o funciones. ¿Qué resultados se desea obtener de tal enseñanza y qué cualidades, en el sentido más amplio de la palabra, se espera desarrollar en los estudiantes? (pp. 11-12).

Ahora bien, sin duda alguna, los métodos deductivos e inductivos son parte de la enseñanza actual del Derecho y, los mismos, se encuentran inmersos en los que son algunos de los 
DERECHO COMPARADO EN INVESTIGACIÓN Y ENSEÑANZA DE DERECHO EN CARRERAS ECONÓMICO ADMINISTRATIVAS.

principales métodos contemporáneos de enseñanza del Derecho; por lo que no es algo nuevo, de hecho, el primer volumen del Yale Law Review, la revista jurídica que publican los estudiantes de Derecho de Yale, en su número 4, data del mes de marzo de 1982, comienza con el título "Los métodos de estudio de le enseñanza del Derecho" (Böhmer, 1999, p.19), de ahí la importancia de definir un método que sea efectivo cuando de la enseñanza del derecho se trata.

Resalta el método de "estudio de Derecho Comparado" (Fix-Zamudio, 2013a, p. 83), técnica para identificar las diferencias y similitudes entre un país perteneciente al mismo sistema de familias jurídicas y un país de diferente familia jurídica. Pérez Lledó (2006) analiza el utilizar lo funcional de los modelos de países de tradiciones jurídicas distintas, como lo es, Estados Unidos y España, donde se anima que en la educación debe incluirse capacidades y destrezas de personas para que apliquen principios y tareas de ponderación y argumentación.

La influencia de otros países permea el derecho doméstico, y es que todo ordenamiento jurídico se ha forjado a través de la influencia de otros sistemas jurídicos, con los cuales se encuentra en contacto en forma permanente, de tal manera que los principios esenciales del derecho desbordan las fronteras políticas (Fix-Zamudio, 2013, p. 2).

También Fix-Zamudio (2013) declara que el Derecho Comparado, "es el sector del conocimiento que estudia la comparación de los ordenamientos jurídicos, y lo define como el instrumento del conocimiento del derecho, y por lo tanto un método jurídico" (p. 28). Por otro lado, también afirma que se tiene la convicción de que "si bien el Derecho Comparado no constituye una panacea para elevar el nivel científico de nuestros estudios jurídicos, en los cuales predomina la información sobre la formación" (Fix-Zamudio, 1973, pp. 23-27), al menos puede contribuir en una proporción apreciable a que los juristas mexicanos se incorporen en mayor número a las nuevas corrientes que se observan en las ciencias jurídicas de nuestra época, "que pretenden contribuir en forma más eficaz a la resolución de los problemas surgidos de un mundo en constante transformación" (Friedmann,1996, pp. 25).

El maestro Fix-Zamudio (2013b) menciona en la obra antes citada, que entre los fines del Derecho Comparado se encuentran:

- La obtención del verdadero nivel científico de los estudios jurídicos, en virtud de que la función esencial del Derecho Comparado es devolver al derecho el carácter universal de 
DERECHO COMPARADO EN INVESTIGACIÓN Y ENSEÑANZA DE DERECHO EN CARRERAS ECONÓMICO ADMINISTRATIVAS.

toda ciencia, pues entre todas las disciplinas científicas, sólo el derecho ha creído falsamente que podía ser puramente nacional.

- Mejor conocimiento nacional.

- Perfeccionamiento del lenguaje jurídico.

- Comprensión internacional del Derecho.

- Unificación o armonización de los ordenamientos jurídicos, y

- Conocimientos dinámicos de los ordenamientos jurídicos (p. 28).

Nos encontramos que "en la comunidad internacional rigen en cierto modo la manera de gobernar de los países, se dice lo anterior, debido a que los cambios jurídicos que se generan en otros países, tarde o temprano, se implementarán en los demás" (Flores Sánchez, 2016, pp. 355-361).

En este sentido, Fix-Zamudio (2013) afirma que:

No podemos aislarnos en nuestro ordenamiento y pretender resolver las cuestiones jurídicas con instrumentos exclusivamente nacionales, sin tomar en cuenta que estamos inmersos en un mundo en el cual se perciben con creciente, pero variable intensidad, las influencias y los contactos con los sistemas jurídicos más diversos que lentamente se aproximan en forma recíproca, y una de las maneras más eficaces de lograr esta comprensión es precisamente el Derecho Comparado (p. 26).

Del Derecho Comparado se debe estudiar desde los logros que el sistema ha tenido en esos países, principales problemas para el éxito de los cambios, (voluntad política, debilidad de los poderes judiciales, resistencia al cambio, deficiencias en la implementación, desatención de ciertos elementos de la reforma, entre otros); así como las estrategias que lograron ese cambio, y el análisis crítico de la situación actual que impera en esos estados extranjeros.

De acuerdo con su desarrollo actual, menciona el profesor emérito Fix-Zamudio (2013b), se puede afirmar que el Derecho Comparado comprende tres sectores esenciales:

a) Exposición del derecho extranjero, el cual constituye el antecedente necesario para la comparación jurídica; es decir, la base sobre la que debe hacerse la comparación.

b) Análisis de los problemas metodológicos de la comparación jurídica, que puede considerarse como el objeto propio de la ciencia del Derecho Comparado en sentido estricto. 
DERECHO COMPARADO EN INVESTIGACIÓN Y ENSEÑANZA DE DERECHO EN CARRERAS ECONÓMICO ADMINISTRATIVAS.

c) Estudio de las disciplinas comparativas de carácter específico, que comprende también el examen de instituciones jurídicas (pp. 37-38).

No se puede dejar a un lado la comparación dentro del derecho nacional; es decir, los distintos ordenamientos dentro de las entidades federativas, dado que, asevera el Dr. Fix-Zamudio:

Sólo cuando se posee un concepto, así sea aproximado, del ordenamiento nacional, que tome en cuenta los factores que se han citado, resulta posible intentar una confrontación con otro u otros sistemas jurídicos, pues sin esta base elemental, el ensayo de comparación resultará incompleto y los resultados pueden ser engañosos o inclusive equivocados, ya sea cuando se realizan intentos de esta naturaleza, frecuentemente se examinan similitudes y diferencias aparentes.

Así, por ejemplo, diferentes Estados de la república o congresos locales legislan materias, por poner un ejemplo, civil o familiar, con criterios muy diferentes, aunque la tendencia actual es la unificación de códigos nacionales como ha ocurrido en materia procesal penal.

Al desarrollar un estudio dentro del Derecho Comparado debemos de tomar en cuenta desde luego, las distintas realidades de diferentes países y evitar errores al implementar cambios dentro de un sistema jurídico.

El Derecho Comparado es entonces "el estudio de las relaciones entre órdenes e instituciones jurídicas y la construcción de doctrina aplicable a las instituciones jurídicas y la construcción de doctrina aplicable a las instituciones jurídicas propias" (Pérez y Cantoral, 2016, p. 41). No se trata de reproducir las leyes de otras latitudes, sino de estudiarlas por medio de este método de estudio que concatenado con otros métodos ayuda a la formación jurídica eficiente de un país.

Si bien, el Derecho Comparado desde luego no constituye el único instrumento para la correcta comprensión del ordenamiento jurídico nacional; sin embargo, "nos puede servir como un sólido apoyo para lograr el equilibrio entre estas tendencias fluctuantes del nacionalismo o de la extranjerización en el campo del derecho, ya que sólo a través de este equilibrio se puede construir una ciencia jurídica verdadera" (Fix-Zamudio, 2013b, pp. 59-60).

Este método de estudio es el que en la actualidad permea de manera significativa dentro del estudio de los Derechos Humanos, Derecho del Trabajo y en la protección jurídica del medio ambiente. Todo lo anterior, tomando en cuenta que al analizar el contexto actual, nos 
DERECHO COMPARADO EN INVESTIGACIÓN Y ENSEÑANZA DE DERECHO EN CARRERAS ECONÓMICO ADMINISTRATIVAS.

encontramos que vivimos en una comunidad globalizada, y hoy más que nunca quedan pocas personas que pueden ser consideradas como extraños en el mundo, (Sen Amartya, 2009, p. 171-173).

La ciencia genómica estableció, "que no hay ninguna base biológica en la que se sustente el concepto de raza" (Alonso, 2013, p. 98), por lo cual, se proclama una igualdad ante la ley y la justicia.

Por tanto, siguiendo el consejo del Dr. Fix-Zamudio, dictado en su discurso de ingreso al Colegio Nacional, en el sentido de que deben incrementarse en forma sustancial los estudios jurídicos comparativos en nuestro país, para lograr la superación de la ciencia jurídica en México que se ha mantenido estática en varios sectores. Entonces, los estudios de Derecho Comparado deben efectuarse a diversos niveles, y para que puedan desarrollarse ampliamente deben iniciarse en la enseñanza, a través de cursos básicos, los cuales se están imponiendo en las Facultades de Derecho en nuestra época, y se agrega que deben realizarse en las diferentes facultades de las diversas licenciaturas en las que se impartan materias de Derecho.

\section{RESULTADOS}

En la investigación empírica realizada, se encontró que son pocos los estudiantes de nivel licenciatura que se interesan en realizar investigaciones de su área (ver figura 1), de 300 estudiantes, solo seis se titulan por artículos científicos, 17 por tesis, y sólo uno por resolución de problemas o casos prácticos. La gran mayoría no realiza investigaciones por no saber efectuarlas.

Conocer y utilizar los métodos y las técnicas de enseñanza contemporáneos del Derecho, ayudarán a realizar investigaciones concretas y aportar tanto a la academia como a la enseñanza del Derecho.

Al entrevistar a profesores que imparten materias del área de Derecho en licenciaturas de Contaduría Pública, Administración, Economía o Mercadotecnia, los métodos que más conocen o utilizan son: Derecho Comparado, Estudio de Caso, Análisis Económico del Derecho, Historia Crítica, Doctrina Analítica.

Se encontró también que son pocos los estudiantes que deciden titularse por medio de investigaciones que concluya en publicación de artículos o tesis. 
DERECHO COMPARADO EN INVESTIGACIÓN Y ENSEÑANZA DE DERECHO EN CARRERAS ECONÓMICO ADMINISTRATIVAS.

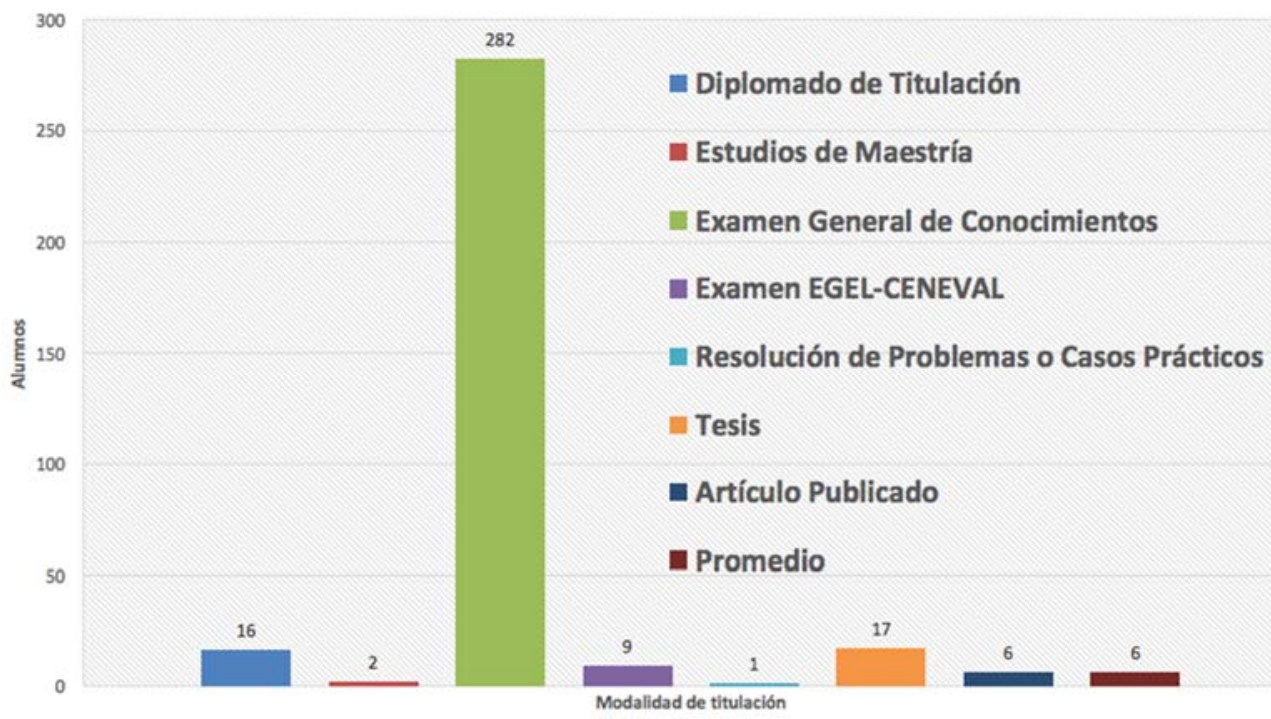

Figura 1. Estudiantes de nivel licenciatura que se interesan en realizar investigaciones.

Fuente: Universidad Juárez Autónoma de Tabasco, División Académica de Ciencias Económico Administrativas.

\section{CONCLUSIONES}

Es de importancia fomentar desde el comienzo en estudiantes universitarios el gen de investigar, de realizar búsquedas por muy sencillas o complejas que parezcan; sin embargo, los profesores universitarios se encuentran en el aula con estudiantes que no saben o no quieren investigar o leer libros. Se les facilita y tienen como costumbre descargar de Internet trabajos y presentaciones ya elaborados, sin cerciorarse del contenido y del lugar donde se generó la información, dado que en ocasiones son de otros países, no de México.

De ahí, que sea deber de los profesores formar generaciones cada vez mejores, se debe usar la tecnología en aprender y aportar en bien de la sociedad.

La calidad de cualquier institución académica depende en definitiva de sus profesores, que son los que en gran medida realizan el plan de estudios, los que enseñan en las aulas, y quienes motivan o enseñan a sus estudiantes a realizar investigaciones. También es necesario puntualizar la gran ayuda que ofrece una buena biblioteca en la universidad, o tener acceso a bases de datos donde pueden consultarse en línea libros y artículos actualizados. En materia de Derecho, diversas editoriales ofrecen el servicio de consulta en línea de su vasta productividad académica basada en libros. 
DERECHO COMPARADO EN INVESTIGACIÓN Y ENSEÑANZA DE DERECHO EN CARRERAS ECONÓMICO ADMINISTRATIVAS.

\section{REFERENCIAS BIBLIOGRÁFICAS}

Alonso, M. (2013). La idea de la vida desde la genética contemporánea. En González J. y Linares J., Diálogos de bioética. Nuevos saberes y valores de la vida, 98. México: Fondo de Cultura Económica UNAM.

Baumman, Z. (2003). Modernidad líquida. México: Fondo de Cultura Económica.

Baumman, Z. (2017). La globalización. Consecuencias Humanas. México: Fondo de Cultura Económica.

Böhmer, M. (1999). La enseñanza del Derecho y el ejercicio de la abogacía. Barcelona: Gedisa.

Eisenmann, C. (2013). Los objetivos y la naturaleza de la enseñanza del Derecho. En Witker J., Antología de estudios sobre enseñanza del Derecho. México: Ediciones Coyoacán.

Constitución Política de los Estados Unidos Mexicanos. (1917). Diario Oficial de la Federación.

Fix-Zamudio, H. (1973). La docencia en las Facultades de Derecho. En 5 Conferencia de las Facultades y Escuelas de Derecho de América Latina. Córdoba: Argentina.

Fix-Zamudio, H. (2013). Algunas reflexiones sobre la enseñanza del Derecho en México y Latinoamérica. En Witker, J., Antología de estudios sobre enseñanza del Derecho, 83. México: Ediciones Coyoacán.

Fix-Zamudio, H. (2013). Derecho Comparado y la ciencia jurídica en México. México: El Colegio de México.

Flores, J. (2016). Familias del siglo XXI, una mirada desde el Derecho. En Díaz, R., Oportunidades para la Investigación e Innovación. Publicaciones Universidad de Montemorelos, 355-361.

González, J. (1998). La construcción del Derecho. Métodos y técnicas de investigación. México: UNAM.

Pérez, G. y Cantoral, K. (2016). Retos de la investigación jurídica en los Posgrados de Calidad: mitos que conspiran en contra. En Pérez G., Temas Actuales de Estudios Jurídicos. México: Tirant Lo Blanch.

Popper, K. (2010). Después de la sociedad abierta. México: Editorial Paidós.

Sen, A. (2009). The Idea of Justice. Cambridge: Harvard University Press.

Witker, V. (2013). Antologías de estudios sobre enseñanza del Derecho. México: Ediciones Coyoacán.

FLORES-SÁNCHEZ J. A., MAGLIONI-MONTALVO R.

ENERO-ABRIL 2019. Año 25, Número 71, Págs. 136-147 